\title{
PSICOEDUCACIÓN GRUPAL CON MENORES INMIGRANTES NO ACOMPAÑADOS.
}

\author{
JUAN MANUEL HERRERA HERNÁNDEZ \\ MARI CARMEN BARRANCO EXPÓSITO \\ NIEVES ROSVERIZ LORENZO MACHÍN \\ NAYRA GONZÁLEZ RODRÍGUEZ \\ RAQUEL HERNÁNDEZ GARCÍA \\ E.U. Trabajo Social. Universidad de la Laguna. Tenerife.
}

\section{MARCO DE REFERENCIA DE LA INTERVENCIÓN.}

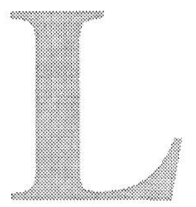

as experiencias sociales o de grupo influyen singularmente en nuestro desarrollo. Se deduce que poseen un gran potencial, si son adecuadamente dirigidos y ofrecen un instrumento fundamental y poderoso de tratamiento. Un grupo de personas puede servir como herramienta terapéutica específica, ya que dentro del mismo, a los participantes se les ofrece de manera singular un variado conjunto de relaciones interpersonales que, con la guía apropiada, les permite identificar, explorar y alterar la conducta interpersonal desadaptada y/o mejorar su calidad de vida ante una dependencia.

Los adolescentes del Hogar del Portezuelo son inmigrantes con los que hay que desarrollar un proceso de integración y adaptación cultural, siempre y cuando no se decida otra cosa por parte de las instituciones pertinentes: Dirección General del Menor y la Familia, la Unidad de Infancia y Familia del Excmo. Cabildo Insular de Tenerife, así como la reciente aprobada Ley de Extranjería.

La integración es el proceso de interrelación de dos sectores culturales que adaptándose mutuamente mantienen sus propias particularidades culturales. La integración supone la capacidad de confrontar y de intercambiar valores, formas, modelos de comportamiento, tanto por parte del inmigrado como de la sociedad receptora. Es un proceso gradual por el cual los nuevos residentes llegan a ser participantes activos en la vida económica, social, cívica, cultural y espiritual del país de inmigración. 
Los niveles de integración van desde la exclusión y la marginación social a la total implicación en la toma de decisiones, desde la postura de "usar" los recursos sociales para ejercer libremente los derechos básicos hasta la postura de "gestionar" los asuntos sociales, tomar parte de las decisiones que inciden en el desarrollo de una sociedad y ser protagonistas de la historia y no meros espectadores.

Trabajar para la integración de las personas inmigrantes, significa favorecer su participación social para analizar el contexto, las situaciones y los conflictos, para desarrollar propuestas que supongan avances cualitativos, para generar una sociedad intelectual, o sea, basada en la relación y no en el aislamiento, para, en resumen, crear nuevas formas sociales, nuevos modos de relación.

Por tanto, la integración también debe significar propiciar que las personas adquieran o desarrollen su capacidad real para lograr cambios sociales. Es por ello, que otra condición para la integración es la interculturalidad, concebida como la relación que se produce entre diversas culturas en el mismo espacio y en un mismo tiempo.

Por todo ello, abordar la problemática de los menores a través de un contexto grupal ha favorecido la mejora de la calidad de vida de los mismos, así como el entrenamiento en competencia social, para facilitarles su pleno desarrollo personal y mejorar sus recursos interpersonales.

\section{OBJETIVOS.}

\subsection{Generales.}

- Intervenir con los adolescentes dentro del grupo para promover la prevención de la violencia y exclusión social.

- Implicar a los profesionales del Centro que mantienen contacto con el menor, y a los profesionales de la Unidad, que participen en el proceso de intervención.

- Promover la normalización de las relaciones interpersonales del menor en su vida cotidiana.

\subsection{Específicos.}

a. Con los menores:

- Ayudar al menor al reconocimiento y valoración de su propia situación de riesgo.

- Educar sobre el sentido del ocio y el tiempo libre. 
- Reforzar los objetivos propuestos en el Proyecto Educativo Individual de cada menor.

b. Con los/as educadores:

- Ayudar al personal educativo a encontrar fórmulas propias de elaboración de soluciones ante los conflictos y en la interacción diaria con el menor.

- Promover en el personal la utilización de instrumentos de registro que faciliten la intervención con los menores.

\subsection{Operativos.}

- Que se observen procesos de cambio en los criterios por los que se decidió su inclusión en el grupo.

- Verificar que el grupo está complementando y apoyando la consecución de objetivos personales.

- Desarrollar una evaluación continua del proceso y resultados conjuntamente: adolescentes, expertos y profesionales de la Unidad.

\section{METODOLOGÍA.}

Todas las acciones que se han ejecutado en dicho proyecto se han basado en una metodología activa, que ha posibilitado la puesta en marcha del mismo y el alcance de los objetivos previstos. Por ello, las actuaciones de los profesionales han jugado un papel decisivo en su puesta en funcionamiento, tanto los profesionales del Centro como de otros proyectos que colaboran en él.

La preparación de los participantes para las dinámicas de grupo ha sido llevada a cabo por el personal del Centro, informando y motivando a los participantes de los beneficios por la asistencia a las sesiones. Esta preparación previa al grupo disminuye los abandonos, aumenta la cohesión y acelera la labor del trabajo grupal.

El espacio de encuentro ha estado precedido por un lugar propicio para la privacidad y el desenvolvimiento adecuado de las dinámicas que se han llevado en las intervenciones grupales. Trabajándose con un grupo reducido de unos 7 jóvenes aproximadamente, se ha podido realizar un trabajo adecuado, pues así se estimaba en la metodología desde un principio acordada.

La duración óptima de una sesión de grupo osciló entre 60 y 90 minutos, pues, generalmente se necesitaban unos 20 ó 30 minutos para el calentamiento, y como mínimo unos 60 minutos para trabajar los temas principales de la sesión. 
El número de sesiones estimado para trabajar fue de una semanal.

El éxito de los grupos no depende de un rígido seguimiento de unas técnicas psicoterapéuticas dadas, sino del estilo de conducción grupal y de la interacción de los miembros del grupo, con la capacidad de aceptación al grupo y no de éste a los profesionales que trabajan dichas intervenciones.

La intervención se ha llevado a cabo por los expertos en intervención grupal con participación activa (presencial) de los profesionales del Centro durante las sesiones.

Este proyecto de intervención con grupos requirió un sistema de coordinación interna entre los profesionales que intervinieron y el personal del Centro y de la Unidad de Infancia y Familia, que posibilitó la consecución de los objetivos generales establecidos. Esta coordinación se ha establecido mediante reuniones informativas y sesiones clínicas.

Para facilitar la toma de decisiones durante el proceso de ejecución se realizó una sesión de coordinación semanal.

De forma paralela a la intervención grupal, se ha llevado a cabo una labor de implicación del personal educativo del Centro, puesto que desarrollar un programa de intervención al margen de los referentes inmediatos del menor, supone perder de vista una importante fuente para promover el cambio y motivación en los menores. Es por ello que el programa ha contado con la implicación del personal, a través de:

- Se ha partido de la motivación y necesidades del personal educativo del Centro para crear un equipo de trabajo con estos profesionales.

- Se ha implicado a este personal en el seguimiento e intervención cotidiana con el menor en el Centro a través del uso de instrumentos evaluativos propios y de su contribución en la evaluación del caso.

- Se han realizado reuniones periódicas con el grupo de personal educativo de centros para valorar la marcha de la intervención.

- Se han mantenido reuniones periódicas de carácter evaluativo y formativo con el personal voluntario que forme el equipo de trabajo.

El equipo de intervención que atendió al grupo de menores estaba formado por terapeutas y co-terapeutas, que intervinieron conjuntamente en las sesiones grupales psicoeducativas, así como dos alumnas de tercer curso de Trabajo Social. 


\section{RESULTADOS.}

Se realizaron un total de 24 sesiones, los martes de $16^{\prime} 00$ a $18^{\prime} 00$ horas, desde el 10 de octubre de 2000 al 11 de abril de 2001, en el Hogar. Los menores participantes en las sesiones fueron siete.

En las sesiones se fueron intercalando temas referidos a la violencia, evitación de la provocación, desarrollo de conductas asertivas, técnicas de relajación, habilidades sociales y cognitivas, auto-conocimiento, derechos y deberes, afrontamiento del estrés, etc.

En cada sesión dos observadoras entrenadas registraban y evaluaban, individualmente, el desarrollo de la sesión, haciendo uso del Estadillo de Registro de Sesiones Psicoeducativas (anexo 1), además de lo que los menores aportaban en la evaluación y valoración de cada sesión, como las aportaciones de los terapeutas grupales. El resultado de la evaluación de las sesiones se presenta a continuación, además de tener en cuenta la evaluación del equipo educativo sobre los menores.

En el siguiente cuadro se presenta la media general alcanzada en cada uno de los indicadores evaluativos a evaluar en cada sesión, pudiendo ser la media máxima a alcanzar en cada sesión de 5 (ver anexo 1).

Cuadro 1: Media de puntuación general por indicadores evaluativos.

\begin{tabular}{|l|c|}
\hline EVALUACIÓN & MEDIA \\
\hline Participación & 3,71 \\
\hline Comunicación & 3,71 \\
\hline Roles & 3,71 \\
\hline Normas & 3,25 \\
\hline Cohesión grupal & 3,54 \\
\hline Objetivos & 3,96 \\
\hline Metodología & 4,13 \\
\hline Recursos & 4,08 \\
\hline Asimilación & 3,75 \\
\hline
\end{tabular}

El siguiente cuadro (véase página siguiente) presenta la puntuación alcanzada en cada sesión realizada, donde el máximo a alcanzar en una sesión puede ser de 45 puntos, pues, de los nueve indicadores a evaluar, cada uno puntuaba de 1 al 5 (ver anexo 1 ).

Si analizamos la gráfica 1 y el cuadro 2 , observamos como ha habido una evolución desde las primeras sesiones hasta las finales, en donde en estas últimas se obtiene la máxima puntuación posible en varias ocasiones. Esto indica que los menores se adaptaron a las sesiones y que el grado de confianza creció en el grupo. 
Cuadro 2: Puntuaciones alcanzadas en cada sesión.

\begin{tabular}{|c|c|c|}
\hline TOTAL & ÍNDICE & $\%$ \\
\hline $1^{a}$ Sesión & 26 & $58 \%$ \\
\hline $2^{a}$ Sesión & 26 & $58 \%$ \\
\hline $3^{a}$ Sesión & 26 & $58 \%$ \\
\hline $4^{\mathrm{a}}$ Sesión & 27 & $60 \%$ \\
\hline $5^{\text {a }}$ Sesión & 23 & $71 \%$ \\
\hline $6^{a}$ Sesión & 19 & $42 \%$ \\
\hline $7^{\mathrm{a}}$ Sesión & 36 & $80 \%$ \\
\hline $8^{a}$ Sesión & 31 & $69 \%$ \\
\hline $9^{a}$ Sesión & 36 & $80 \%$ \\
\hline $10^{\mathrm{a}}$ Sesión & 33 & $73 \%$ \\
\hline $11^{\text {a }}$ Sesión & 28 & $62 \%$ \\
\hline $12^{\mathrm{a}}$ Sesión & 33 & $73 \%$ \\
\hline $13^{\mathrm{a}}$ Sesión & 34 & $76 \%$ \\
\hline $14^{\mathrm{a}}$ Sesión & 39 & $87 \%$ \\
\hline $15^{\text {a }}$ Sesión & 27 & $60 \%$ \\
\hline $16^{\text {a }}$ Sesión & 40 & $89 \%$ \\
\hline $17^{\text {a }}$ Sesión & 45 & $100 \%$ \\
\hline $18^{\mathrm{a}}$ Sesión & 39 & $87 \%$ \\
\hline $19^{\mathrm{a}}$ Sesión & 33 & $73 \%$ \\
\hline $20^{\mathrm{a}}$ Sesión & 36 & $80 \%$ \\
\hline $21^{\mathrm{a}}$ Sesión & 45 & $100 \%$ \\
\hline $22^{\mathbf{a}}$ Sesión & 43 & $95 \%$ \\
\hline $23^{a}$ Sesión & 45 & $100 \%$ \\
\hline $24^{\mathrm{a}}$ Sesión & 45 & $100 \%$ \\
\hline Media & 33,96 & $75 \%$ \\
\hline
\end{tabular}

Gráfica 1: Evolución de las puntuaciones en las sesiones.

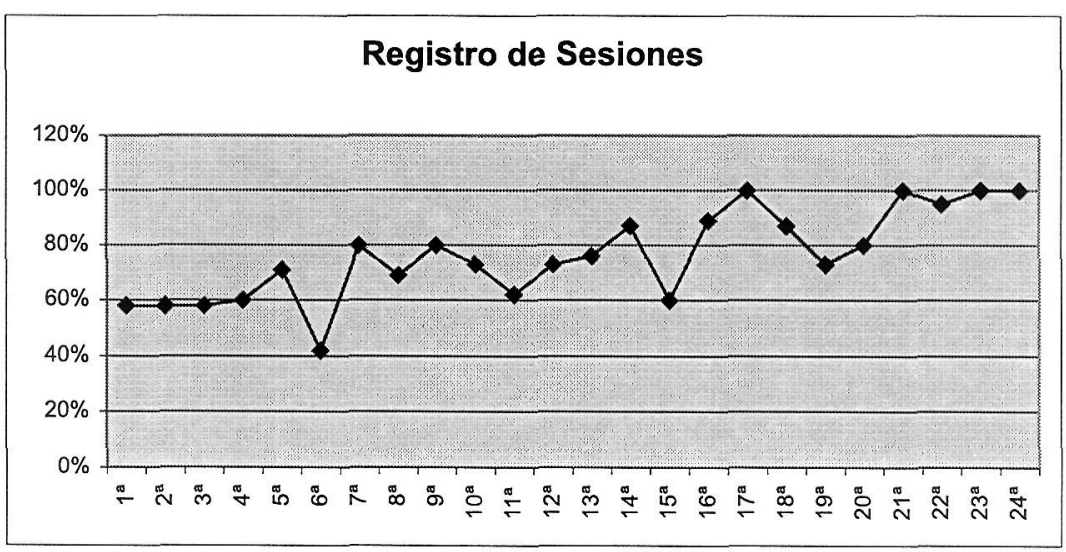


Hemos de tener en cuenta que la línea no es uniforme, ya que las incidencias de la vida cotidiana, en muchas ocasiones, afectaba a la puesta en práctica de las intervenciones.

Hay que resaltar que a lo largo de la intervención no se recibió notificación alguna o no se tuvo constancia, por parte de la policía municipal o nacional, así como de los centros educativos, programas de garantía social o talleres ocupacionales, donde estaban los menores distribuidos, respecto a hechos delictivos, violentos o agresiones hacia otros por parte de los menores inmigrantes.

\section{CONCLUSIONES.}

Prácticamente, en todas las sesiones han asistido la totalidad de los jóvenes del Centro, siete. También es cierto que la no asistencia, en su mayor parte, ha sido por causa justificada (encontrarse enfermo o tener que ir a clase...), por tanto, también, podemos hablar de una mayor motivación e implicación de los chicos a lo largo de las sesiones.

Asimismo, algunos de los temas que se presentaron, como sexualidad, drogas..., han sido muy difíciles de tratar, además de encontrarnos con situaciones inesperadas (como fue la época del Ramadán), por sus valores religiosos y su cultura.

Por esta misma causa se ha intentado que no se llevara a cabo una integración bajo la creación de guetos. Por ello, se llevaron iniciativas de un fomento de relaciones con personas no pertenecientes al Centro ni propias de su cultura, para su integración en la comunidad canaria, así como la puesta en práctica del entrenamiento recibido.

Se practicaron varias reuniones, no sólo semanalmente con los educadores antes y después de cada sesión, sino mensuales, con lo demás proyectos y personal del Centro, para llevar a cabo una coordinación, e incluso realizar actividades conjuntas.

Sobre las sesiones decir que se han valorado positivamente, puesto que nos encontramos con jóvenes que se pueden en su mayoría, integrar en la sociedad canaria, siempre y cuando reciban las condiciones educativas y formativas adecuadas, paralelamente a intervenciones psicoeducativas de esta índole para prevenir una marginación no sólo laboral sino social.

Después de finalizada la intervención grupal, el grupo de menores inmigrantes no acompañados, fue integrado dentro de otro grupo de menores fuera de su Hogar, con características similares, pero no inmigrantes, para facilitar una mayor integración cultural y fomentar la normalización. 


\section{BIBLIOGRAFÍA.}

CAMACHO MARTÍN, V.; DÁVILA DE LEÓN, G.; HERRERA HERNÁNDEZ, J.M.; GONZÁELEZ REYES, M.S.; OLIVERA ROMERO, M.; 1.999: "Proyecto Pa'lante". Tenerife.

FERNÁNDEZ DEL VALLE, J.; FUERTES ZURITA, J.; 2.000: “El acogimiento residencial en la protección a la infancia". Ediciones Pirámide. Madrid.

POLO RODRÍGUEZ, J.J.; HUÉTAMO BUENDÍA, A.J.; 2.000: "La nueva Ley Penal del Menor". Editorial COLEX. Madrid.

RUTTER, M.; GILLER, H.; HAGELL, A.; 2.000: "La conducta antisocial de los jóvenes". Editorial Cambridge. Madrid.

VV.AA.; 2.000: "Intervención en inadaptación social". Editorial Síntesis Educación. Madrid.

\section{ANEXO 1:}

ESTADILLO DE REGISTRO DE SESIONES PSICOEDUCATIVAS GRUPALES. Herrera Hernández, J.M. (1999).

- N. sesión:

- Fecha: ......... de

- Lugar:

- Hora: de

- Terapeuta:

- Coterapeuta:

- Observadores:

OBJETIVOS:

$1 .^{\circ}$

$2 .^{\circ}$

3. ${ }^{\circ}$

METODOLOGÍA:

RECURSOS:

- N. ${ }^{\circ}$ de asistentes:

- N. ${ }^{\circ}$ chicos:

- Otroa profesionales:

\section{RECURSOS:}


Psicoeducación grupal con menores inmigrantes no acompañados. 377

EVALUACIÓN

\begin{tabular}{|l|l|l|l|l|l|}
\hline Participación & 1 & 2 & 3 & 4 & 5 \\
\hline Comunicación & 1 & 2 & 3 & 4 & 5 \\
\hline Roles & 1 & 2 & 3 & 4 & 5 \\
\hline Normas & 1 & 2 & 3 & 4 & 5 \\
\hline Cohesión Grupal & 1 & 2 & 3 & 4 & 5 \\
\hline Objetivos & 1 & 2 & 3 & 4 & 5 \\
\hline Metodología & 1 & 2 & 3 & 4 & 5 \\
\hline Recursos & 1 & 2 & 3 & 4 & 5 \\
\hline Asimilación & 1 & 2 & 3 & 4 & 5 \\
\hline GENERAL & & & & & \\
\hline
\end{tabular}

PUNTUACION GENERAL:

OBSERVACIONES:

\begin{tabular}{|l|l|l|l|l|}
\hline 9 & & 27 & & 45 \\
\hline
\end{tabular}

\section{LEYENDA DE LOS INDICADORES DE EVALUACIÓN}

A. Participación (hablan, interactúan, escucha activa, etc,.).

1. Inexistencia de participación.

2. Menos de la mitad del grupo participa.

3. Participa la mitad.

4. Participa más de la mitad.

5. Buena participación grupal (todos).

B. Comunicación (hablar, escuchar, confrontar,etc.).

1. Inexistencia de comunicación (sólo monólogos o respuestas afirmativas o negativas).

2. La comunicación se da en menos de la mitad del grupo.

3. Se da en la mitad del grupo.

4. La comunicación se da en más de la mitad del grupo.

5. Buena comunicación intergrupal (todos).

C. Desempeño de roles (terapeuta, coterapeuta, observadores).

1. No se desempeña ningún rol en la actividad.

2. Se desempeñan roles no asignados o propuestos.

3. Desempeño regular de/los rol/es asignados.

4. Normal desempeño.

5. Buen desempeño de/los rol/es.

D. Cumplimiento de normas (respeto de palabra, volumen de voz, posición sentado, comportamiento...).

1. No se cumplen las normas básicas establecidas.

2. Las cumplen menos de la mitad del grupo.

3. Cumplen las normas la mitad del grupo.

4. Cumplen más de la mitad del grupo las normas.

5. Buen cumplimiento de normas. 
E. Cohesión grupal (unidad grupal, conexión en el grupo, consistencia, coherencia...).

1. Inexistencia de cohesión grupal.

2. Existe pero en menos de la mitad del grupo.

3. Existe con la mitad del grupo.

4. Existe con más de la mitad.

5. Buena cohesión grupal.

F. Consecución de objetivos (referido a los «operativos»).

1. No se inicia la consecución de objetivos.

2. Se alcanza una cuarta parte de los objetivos.

3. Se alcanzan la mitad de los objetivos.

4. Se alcanzan más de la mitad de los mismos.

5. Se alcanzan todos los objetivos.

G. Metodología (técnicas, dinámicas, organización...).

1. No se hace uso de la metodología propuesta.

2. Se hace uso no siendo la acertada.

3. La metodología ayuda poco en el desarrollo de la actividad.

4. Ayuda para el desarrollo de la actividad.

5. La metodología acertada.

H. Recursos (humanos, materiales, técnicos...).

1. No se hace uso de los recursos propuestos.

2. Se utilizan menos de la mitad de los recursos.

3. Se hace uso de la mitad de los recursos.

4. Se utilizan más de la mitad.

5. Se hace uso de todos los recursos.

I. Asimilación (aprehensión, comprensión, propicia reflexión, debate, cambio de opiniones, actitudes, interés, aceptación, recepción...).

1. No se refleja aparentemente asimilación.

2. Escasa asimilación.

3. Algo.

4. Normal

5. Buena. 